\title{
Perimesencephalic Nonaneurysmal Subarachnoid Hemorrhage With Unilateral Third Cranial Nerve Palsy -Two Case Reports-
}

\author{
Nobutake SADAMASA, ${ }^{1}$ Noritaka SANO, ${ }^{1}$ Nobuhiko TAKEDA, ${ }^{1}$ \\ Kazumichi YOSHIDA, ${ }^{1}$ Osamu NARUMI, ${ }^{1}$ Masaki CHIN,${ }^{1}$ and Sen YAMAGATA ${ }^{1}$ \\ ${ }^{1}$ Department of Neurosurgery, Kurashiki Central Hospital, Kurashiki, Okayama
}

\begin{abstract}
Perimesencephalic nonaneurysmal subarachnoid hemorrhage (PNSAH) is a benign form of subarachnoid hemorrhage and is usually not associated with any focal deficit. We describe two rare cases of PNSAH with unilateral third cranial nerve palsy. Both patients were treated conservatively. The outcomes of our two cases were excellent. The third cranial nerve palsy resolved gradually in both patients, suggesting that the prognosis for third cranial nerve palsy concomitant with PNSAH is favorable. Unilateral third cranial nerve palsy may occur as the first clinical manifestation of PNSAH.
\end{abstract}

Key words: subarachnoid hemorrhage, third cranial nerve palsy

\section{Introduction}

Subarachnoid hemorrhage (SAH) of unknown origin, defined as no demonstrable lesion revealed by repeated angiography, accounts for about $15 \%$ of cases. ${ }^{7)}$ Perimesencephalic nonaneurysmal SAH (PNSAH) is one of the most common patterns of SAH of unknown origin, characterized by the unique distribution of subarachnoid hematoma confined to the cisterns around the midbrain. ${ }^{6)}$ Generally, the clinical manifestation of PNSAH is sudden onset of headache, similar to that of aneurysmal SAH. ${ }^{8)}$ The natural history of PNSAH is benign, and PNSAH causing focal neurological deficit such as unilateral cranial nerve palsy is quite rare. Recently, the prevalence of anosmia was reported as one in 16 of patients with PNSAH. ${ }^{2)}$

Unilateral third cranial nerve palsy is a clinical manifestation of ruptured and unruptured internal carotid-posterior communicating artery aneurysm which compresses the oculomotor nerve. Indirect compression by uncal herniation with increased intracranial pressure (ICP) after the rupture of cerebral aneurysms in the anterior circulation also results in unilateral third nerve palsy. However, PNSAH does not usually cause increased ICP and does not have any compressive component such as aneurysms. Only one case of PNSAH associated with unilateral third cranial nerve palsy has been reported.

We describe two rare cases of PNSAH manifesting as third cranial nerve palsy, and discuss the cause of the palsy in these and previous cases of PNSAH with cranial palsies.

Received July 28, 2011;

Accepted February 24, 2012

\section{Case Reports}

Case 1: A 63-year-old male was admitted to our hospital because of sudden right ptosis, photophobia, and diplopia. He had a history of hypertension, diabetes, and tuberculosis. On admission, his consciousness was alert and oriented. The right pupil was dilated, both direct and indirect light reflexes were absent, and the right eyeball was deviated laterally with complete absence of adduction. The right pupil was $4 \mathrm{~mm}$ in diameter; the left pupil was $2 \mathrm{~mm}$ in diameter. No other neurological deficit was found.

Head computed tomography (CT) on admission showed $\mathrm{SAH}$ in the interpeduncular and ambient cisterns (Fig. 1A). Subsequent three-dimensional CT with contrast medium and digital subtraction angiography (DSA) showed no apparent bleeding source (Fig. 1B). Therefore, the diagnosis was PNSAH. Diffusion-weighted magnetic resonance (MR) imaging on the same day revealed high intensity in the right midbrain and pons, indicating acute ischemic lesion, but no thrombosed aneurysm (Fig. 1C). The patient was treated conservatively. DSA was re-performed on day 10, and showed no apparent bleeding source (Fig. 1D, E). His right third cranial nerve palsy gradually improved. The light reflex in the right pupil returned on day 4 , and diplopia disappeared on day 8 . The patient was discharged on day 22 without abnormal findings on head CT (Fig. 1F) and no neurological deficit was observed during the follow-up period.

Case 2: A 66-year-old male was admitted to our hospital because of mild headache and sudden left ptosis. He had a history of hypertension, diabetes, and chronic renal failure, and needed hemodialysis. On admission, his consciousness was alert and oriented. The left pupil was dilated, both direct and indirect light reflexes were preserved, 

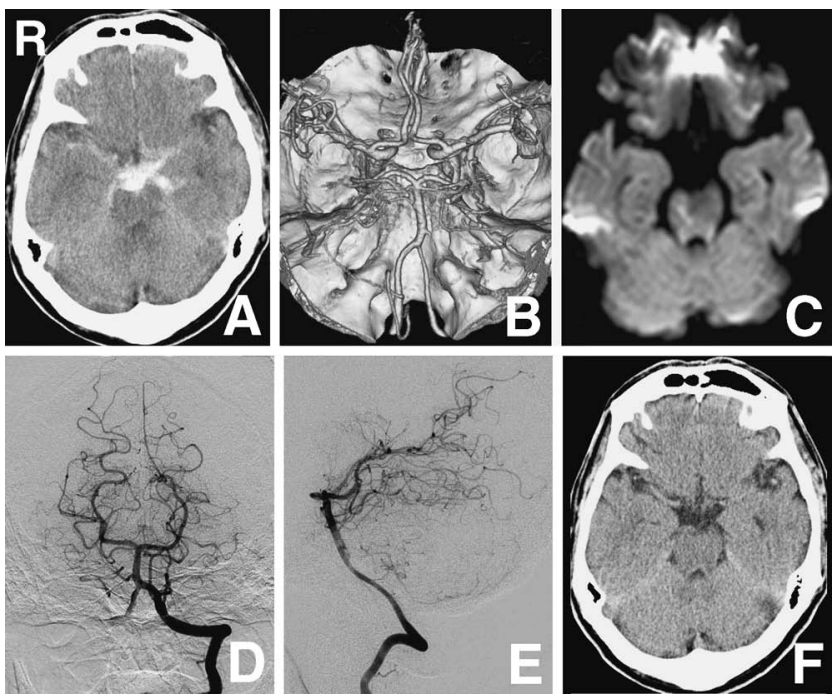

Fig. 1 Case 1. A: Head computed tomography (CT) scan on admission showing subarachnoid hemorrhage mainly distributed around the midbrain and pons. B: Subsequent CT angiogram indicating no apparent bleeding source. C: Diffusionweighted magnetic resonance image on admission showing hyperintensity in the right pons. D, E: Digital subtraction angiograms including vertebral arteriogram on day 10 showing no apparent bleeding source. F: Follow-up CT scan on discharge showing no abnormal finding.
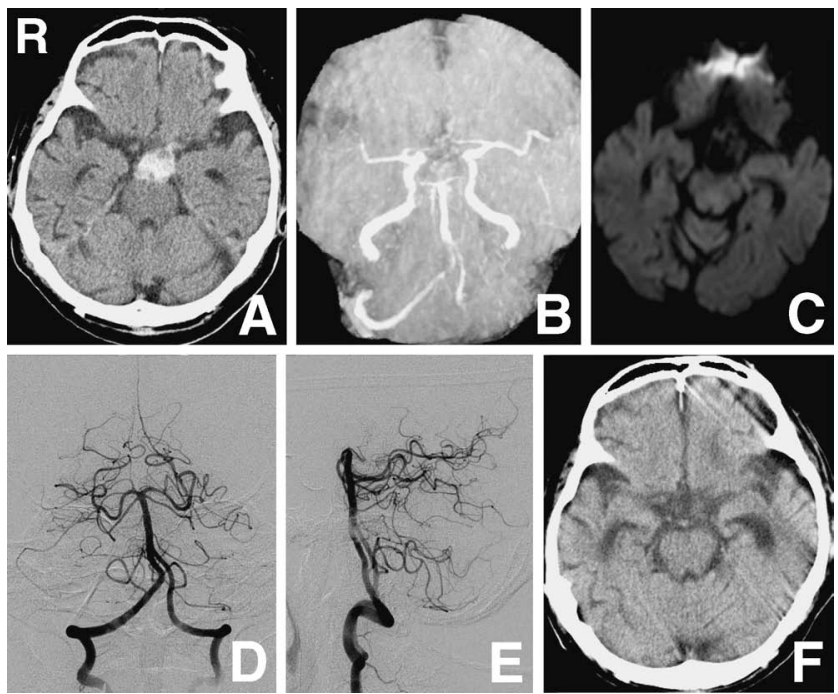

Fig. 2 Case 2. A: Head computed tomography (CT) scan on admission showing subarachnoid hemorrhage distributed around the pons. B: Subsequent magnetic resonance angiogram indicating no apparent bleeding source. C: Diffusionweighted magnetic resonance image on admission showing no hyperintensity. D, E: Digital subtraction angiograms including vertebral arteriogram showing no apparent bleeding source. F: Follow-up CT scan after 6 months showing no abnormal finding. and the left eyeball was deviated laterally with complete absence of adduction. The left pupil was $4 \mathrm{~mm}$ in diameter; the right pupil was $2 \mathrm{~mm}$ in diameter. No other neurological deficit was found.

Head CT on admission showed SAH in the interpeduncular and prepontine cisterns (Fig. 2A). Subsequent MR angiography and DSA showed no apparent bleeding source (Fig. 2B, D, E), and the diagnosis was PNSAH. Diffusion-weighted MR imaging on the same day revealed no high intensity lesion in the brainstem, and no thrombosed aneurysm (Fig. 2C). The patient received conservative therapy. DSA could not be performed again, because of the patient's refusal. After admission, both direct and indirect light reflexes in his left pupil became absent on day 2. The symptoms stayed the same until discharge. The patient was discharged on day 5 . On day 12 , ptosis and diplopia were still observed. On day 44 , he had no complaint in the eye. Head CT showed no abnormal finding at follow-up examination after 6 months (Fig. 2F).

\section{Discussion}

Unilateral third cranial nerve palsy is rare as the first clinical manifestation of PNSAH. Ten of 127 patients with $\mathrm{SAH}$ of unknown origin presented with focal neurological deficits, 5 with hemiparesis, 2 with leg paresis, 1 with positive Babinski reflex, 1 with facial nerve palsy, and 1 with both abducens and facial nerve palsy. ${ }^{1)}$ Headache was only the first symptom in 65 cases of PNSAH. ${ }^{5}$ Only one previous case of PNSAH with unilateral third cranial nerve palsy was reported in 2005. ${ }^{3)}$

No bleeding source that could directly compress the third cranial nerve was identified in our two cases, so three mechanisms are possible to explain how the cranial nerve palsy developed after PNSAH: indirect compression by the hematoma, focal ischemia by occlusion or collapse of a perforating artery, and vasospasm. In the previous case, MR angiography showed that the basilar artery was spastic on day 3 , so the oculomotor palsy seemed to be due to vasospasm. ${ }^{3)}$ Our Case 1 showed no vasospasm on angiography, but initial diffusion-weighted imaging showed hyperintensity in the brainstem including the midbrain, so the cause of the third cranial nerve palsy might be focal ischemia caused by occlusion or collapse of the perforating artery. Concurrent focal ischemia, including delayed ischemic neurological deficit, in patients with PNSAH is rare, and only $1-5 \%$ of the patients with PNSAH revealed symptomatic vasospasm.7)

In our Case 2, no apparent vasospasm was detected by angiography and no acute ischemic lesion was found on initial MR imaging. The hematoma was located mainly on the left, so the left third cranial nerve palsy might have been caused by indirect compression or extension by the hematoma. A previous case of SAH caused by ruptured superior cerebellar artery aneurysm was followed by left ptosis on day $3 .{ }^{4)}$ The unilateral third cranial nerve palsy could result from three possible causes: local toxic degradation products from the hematoma, ischemia of the vessel that feeds the nerve, or compression by overextended arachnoid space. One or more of these three factors might 
have been involved in the development of the third cranial nerve palsy in Case 2.

Pupil-sparing third cranial nerve palsy is thought to be the result of extraaxial microvascular ischemia and is usually observed in diabetic patients. In our Case 2 with diabetes, the light reflex of the left pupil was preserved on admission, suggesting that this pupil-sparing oculomotor palsy might be partly due to diabetic vasculopathy. SAH would enhance the damage to the nerve in the acute stage. The recovery from the symptom was slower in Case 2 in spite of clearance of the subarachnoid clot, possibly due to diabetic vasculopathy. His clinical course with acute aggravation and slow recovery implied that both diabetes and the subarachnoid clot might have been factors in the third cranial palsy.

The outcomes of our two cases were excellent and consistent with that previously reported for PNSAH.7) The third cranial nerve palsy resolved gradually in both patients, suggesting that the prognosis for third cranial nerve palsy concomitant with PNSAH is favorable. Therefore, we should keep in mind the possibility that unilateral third cranial nerve palsy is the first clinical manifestation of PNSAH.

\section{Disclosure}

The authors report no conflict of interest concerning the materials or methods used in this study or the findings specified in this paper.

\section{References}

1) Brismar J, Sundbarg G: Subarachnoid hemorrhage of unknown origin: Prognosis and prognostic factors. J Neurosurg 63: 349-354, 1985

2) Greebe P, Rinkel GJE, Algra A: Anosmia after perimesencephalic nonaneurysmal hemorrhage. Stroke 40: 2885-2886, 2009

3) Kamat AA, Tizzard S, Mathew B: Painful third nerve palsy in a patient with perimesencephalic subarachnoid haemorrhage. Br J Neurosurg 19: 247-250, 2005

4) Meyer YJ, Paine JT, Batjer HH: Focal subarachnoid hematoma: an unusual cause of delayed third cranial nerve paralysis. Surg Neurol 34: 169-172, 1990

5) Rinkel GJ, Wijdicks EF, Vermeulen M, Hasan D, Brouwers PJ, van Gijn J: The clinical course of perimesencephalic nonaneurysmal subarachnoid hemorrhage. Ann Neurol 29: 463-486, 1991

6) Rinkel GJ, Wijdicks EF, Vermeulen M, Ramos LM, Tanghe HL, Hasan D, Meiners LC, van Gijn J: Nonaneurysmal perimesencephalic subarachnoid hemorrhage: CT and MR patterns that differ from aneurismal rupture. AJNR Am J Neuroradiol 12: 829-834, 1991

7) Schwartz TH, Solomon RA: Perimesencephalic nonaneurysmal subarachnoid hemorrhage: review of the literature. Neurosurgery 39: 433-440, 1996

8) van Gijn J, van Dongen KJ, Vermeulen M, Hidra A: Perimesencephalic hemorrhage: A nonaneurysmal and benign form of subarachnoid hemorrhage. Neurology 35: 493-497, 1985

Addressreprint requests to: Nobutake Sadamasa, MD, PhD, Department of Neurosurgery, Kurashiki Central Hospital, 1-1-1 Miwa, Kurashiki, Okayama 710-8602, Japan. e-mail: ns11267@kchnet.or.jp 